Pacific Journal of Mathematics

CHAINS OF INFINITE ORDER AND THEIR APPLICATION TO

John W. Lambert and Patrick Colonel SuP 


\section{CHAINS OF INFINITE ORDER AND THEIR APPLICATION TO LEARNING THEORY}

\section{John Lamperti ANd Patrick Suppes}

1. Introduction. The purpose of this paper is to study the asymptotic behavior of a large class of stochastic processes which have been used as models of learning experiments. We will do this by applying a theory of so-called "chains of infinite order" or "chaînes à liaisons complètes." Namely, we shall employ certain limit theorems for stochastic processes whose transition probabilities depend on the entire past history of the process, but only slightly on the remote past. Such theorems were given by Doeblin and Fortet [3] in a form close to that we employ; however, in order to accomodate certain cases of learning models we found it necessary to relax somewhat their hypotheses. A self-contained discussion of these and some additional results is the content of $\S 2$.

We should emphasize that this section is included to serve as preparation for the theorems of $\S 4$, and it is original with us only in some details and extensions. In addition to [3], papers by Harris [7] and Karlin [8] contain very closely related results and arguments, but not quite in the form we require.

The processes which we shall study with these tools are called "linear earning models." From a psychological standpoint these models are very simple. A subject is presented a series of trials, and on each trial he makes a response, which consists of a choice from a finite set of possible actions. This response is followed by a reinforcement (again one of a finite number). The assumption of the model is that the subject's response probabilities on the next trial are linear functions of the probabilities on the present trial, where the form of the functions depends upon which reinforcement has occurred. Many results about such models may be found in Bush and Mosteller [2], Estes [4], and Estes and Suppes [6]. We will also study here models constructed along similar lines for experiments involving two or more subjects and a type of interaction between them [6, Section 9] and Atkinson and Suppes [1]. Precise definitions of these processes are given below in $\S 3$.

The references mentioned above do not, except in very special cases, give a thorough treatment of asymptotic properties. We shall prove that under general conditions linear learning models exhibit "ergodic" behavior; that is, that after much time has passed these processes become approximately stationary and the influence of the initial distributions

Received November 20, 1958. This research was supported by the Group Psychology and Statistics Branches of the Office of Naval Research under contracts with Stanford University. 
goes to zero. This is not the case for all models which have been used in experimental work, but it seems as if ergodic behavior can be proved by our method in almost all the cases in which one might expect it. Our theorems to this effect, their proofs and some corollaries are given in $\S 4$.

The major work so far on limiting behavior of learning models is Karlin [8], who obtains detailed limit theorems for certain classes of models. However, the results and even the techniques of Karlin's paper do not apply to many cases of interest. His starting point is a representation of the linear model as a Markov process whose states are the response probabilities. Two typical situations when such a representation is impractical arise (i) when the probabilities with which the reinforcement is selected depend on two or more previous responses, and (ii) in the many-person situations mentioned above. Both these situations can (and will) be studied using infinite order chains, and ergodic behavior established under mild restrictions. On the other hand, Karlin's work treats interesting non-ergodic cases outside the scope of our approach. For example, consider a $T$-maze experiment in which the subject (a rat, say) is reinforced (rewarded) on each trial regardless of whether he goes left or right. In the appropriate linear model, the probability of a left turn eventually is either nearly 0 or nearly 1 , and which it is depends upon the rat's initial response probabilities. The model of this experiment has been thoroughly studied in [8, Section 2], and these results have been generalized by Kennedy [9].

In conclusion we comment that both more detailed results and other applications seem possible using the ideas of "infinite order chains." We hope to contribute further to this development in the future.

2. Chains of infinite order. In this section we present a theory of non-Markov stochastic processes where the transition probabilities are influenced only slightly by the remote past. The original convergence theorems for this type of process are due to Doeblin and Fortet [3]; they are given here in a generalized form (Theorems 2.1 and 2.2). The weaker hypotheses make the proof of Lemma 2.1 more complicated than it is in [3], but the other proofs are not much affected. T. E. Harris has also studied these chains; we shall not use his results but remark that his paper [7] gives additional references and background on the subject. Finally we point out that the restriction to a finite number of states is not essential, and the theorems can be extended to the denumerable case without much change of methods.

Let $I$ consist of the integers from 1 to $N$ (to represent the states of the chain); we shall use the notation $x$ for a finite sequence $i_{0}, i_{1}, \cdots$ of integers from $I$. The subscript " $m$ " on $x_{m}$ merely adds the specifica- 
tion that the sequence has $m$ terms; the "sum" $x_{m}+x^{\prime}$ will be the combined sequence $i_{0}, \cdots, i_{m-1}, i_{0}^{\prime}, i_{1}^{\prime} \cdots$. The starting point for the theory will be a set of functions $p_{i}(x)$ defined for all $i \in I$ and all sequences $x$ (including the sequence $\rho$ of length zero) and having the properties

$$
p_{i}(x) \geqq 0, \sum_{i} p_{i}(x)=1 .
$$

The function $p_{i}(x)$ will be interpreted as the conditional probability that a path function of the random process will go next to state $i$, having just occupied state $i_{0}$, previously $i_{1}$, etc. With this interpretation in mind we define inductively the "higher transition probabilities":

$$
p_{i}^{(n)}(x)=\sum_{j \in I} p_{j}(x) p_{i}^{(n-1)}(j+x),
$$

where of course $p_{i}^{(1)}(x)=p_{i}(x)$, the given function. It is easy to see that these higher probabilities also satisfy condition (2.1). The functions $p_{i}^{(n)}(x)$ are the analogues of the terms of the matrix $P^{n}$ for a Markov chain with transition matrix $P$; the theorems we shall give generalize the convergence properties of the matrices $P^{n}$.

We shall first impose a positivity condition on the transition probabilities; specifically we assume that for some state $j_{0}$, some positive integer $n_{0}$, and some $\delta>0$,

$$
p_{j_{0}}^{\left(n_{0}\right)}(x)>\delta \text { for every } x .
$$

We also need to make precise the "slight" dependence of these probabilities on the remote past; indeed, this is the crux of the whole theory. Define

$$
\varepsilon_{m}=\sup \left|p_{i}\left(x+x^{\prime}\right)-p_{i}\left(x+x^{\prime \prime}\right)\right|
$$

where the sup is taken over all states $i$, all sequences $x^{\prime}$ and $x^{\prime \prime}$, and all sequences $x$ which contain the state $j_{0}$ at least $m$ times. We shall use the postulate

$$
\sum_{m=0}^{\infty} \varepsilon_{m}<\infty .
$$

(In [3], $\varepsilon_{m}$ is defined in the same way except that the sup is taken over all $x$ of length at least $m$. Since this results in larger $\varepsilon_{m}^{\prime} s$, and since it is also assumed there that $\sum \varepsilon_{m}<\infty$, our hypotheses are strictly weaker.) Throughout this section, (2.3) and (2.5) will be assumed.

LEMMA 2.1.

$$
\lim _{m \rightarrow \infty}\left[\sup \left|p_{i}^{(n)}\left(x+x^{\prime}\right)-p_{i}^{(n)}\left(x+x^{\prime \prime}\right)\right|\right]=0,
$$

where the sup is the same as in (2.4) (i.e., $x$ contains $j_{0}$ at least $m$ times); the convergence is uniform in $n$. 
Proof. We define quantites $\varepsilon_{m}^{(k)}$ by using $p_{i}^{(k)}$ instead of $p_{i}$ in (2.4); then of course $\varepsilon_{m}^{(1)}=\varepsilon_{m}$, and the conclusion of the lemma is equivalent to $\varepsilon_{m}^{(k)} \rightarrow 0$ uniformly in $k$ as $m \rightarrow \infty$. Now

$$
\begin{aligned}
& \left|p_{i}^{(k)}\left(x+x^{\prime}\right)-p_{i}^{(k)}\left(x+x^{\prime \prime}\right)\right| \\
= & \left|\sum_{j}\left\{p_{i}^{(k-1)}\left(j+x+x^{\prime}\right) p_{j}\left(x+x^{\prime}\right)-p_{i}^{(k-1)}\left(j+x+x^{\prime \prime}\right) p_{j}\left(x+x^{\prime \prime}\right)\right\}\right| \\
\leqq & \sum_{j} p_{j}\left(x+x^{\prime}\right)\left|p_{i}^{(k-1)}\left(j+x+x^{\prime}\right)-p_{i}^{(k-1)}\left(j+x+x^{\prime \prime}\right)\right| \\
+ & \sum_{j}\left|p_{j}\left(x+x^{\prime}\right)-p_{j}\left(x+x^{\prime \prime}\right)\right| p_{i}^{(k-1)}\left(j+x+x^{\prime \prime}\right) .
\end{aligned}
$$

Suppose that $x$ contains $j_{0} m$ times. Then the second term of the above estimate is less than $N \varepsilon_{m}$. The absolute value in the first term is less than $\varepsilon_{m}^{(k-1)}$, but if $j=j_{0}$ this can be improved to $\varepsilon_{m+1}^{(k-1)}$. Taking account of (2.3) and assuming that $n_{0}=1$, we obtain the estimate

$$
\varepsilon_{m}^{(k)} \leqq N \varepsilon_{m}+\delta \varepsilon_{m+1}^{(k-1)}+(1-\delta) \varepsilon_{m}^{(k-1)} .
$$

(In case $n_{0}>1$, the same idea can be carried out; the details are more cumbersome and will not be given.)

Now (2.7) can be iterated to obtain an estimate of $\varepsilon_{m}^{(k)}$ in terms of $\varepsilon_{m}$. After some computation the result is

$$
\begin{aligned}
\varepsilon_{m}^{(k)} & \leqq N \varepsilon_{m} \sum_{i=0}^{k-1}(1-\delta)^{i}+N \varepsilon_{m+1} \delta \sum_{i=0}^{k-2}(i+1)(1-\delta)^{l} \\
& +\cdots+N \varepsilon_{m+l} \delta^{l} \sum_{i=0}^{k-l-1}\left(\begin{array}{c}
i+l \\
i
\end{array}\right)(1-\delta)^{i}+\cdots+N \delta^{k-1} \varepsilon_{m+k-1} .
\end{aligned}
$$

If the series are extended to infinity, the inequality remains true; calling these (infinite) series $A_{0}, A_{1}, \cdots, A_{k-1}$ we have

$$
\varepsilon_{m}^{(k)} \leqq N \sum_{i=0}^{k-1} \varepsilon_{m+i} \delta^{i} A_{i}
$$

But it can be shown without much difficulty that

$$
A_{l+1}-A_{l}=(1-\delta) A_{l+1},
$$

or $A_{l+1}=A_{l} / \delta$. Since $A_{0}=\delta^{-1}$ we obtain $A_{l}=\delta^{-(l+1)}$, and hence

$$
\varepsilon_{m}^{(k)} \leqq \delta^{-1} \sum_{i=0}^{k-1} \varepsilon_{m+i}
$$

Recalling hypothesis (2.5), the uniform convergence of $\varepsilon_{m}^{(k)}$ follows from (2.8).

LEMMA 2.2.

$$
\lim _{n \rightarrow \infty}\left|p_{i}^{(n)}\left(x^{\prime}\right)-p_{i}^{(n)}\left(x^{\prime \prime}\right)\right|=0
$$

and the convergence is uniform in $x^{\prime}$ and $x^{\prime \prime}$. 
Proof. For clarity we shall use probabilistic arguments, although a purely analytic rephrasing is not hard. Consider two stochastic processes operating independently with transition probabilities $p_{i}(x)$, one with the sequence $x^{\prime}$ for its past history up to time 0 and the other with $x^{\prime \prime}$. In view of Lemma 2.1, for any $\varepsilon>0$ there is an $m$ such that if the two processes have occupied the same states for a period which includes $j_{0}$ at least $m$ times and ends sometime before time $n$, then their probabilities of being in state $i$ at time $n$ differ by at most $\varepsilon / 2$. But it follows from condition (2.3) that with probability one, there will sometime be a period of length $m$ during which both processes remain in state $j_{0}$. We can take $n$ large enough so that this simultaneous "run" of state $j_{0}$ will occur before time $n$ with probability not less than $1-\varepsilon / 2$. For this and all greater values of $n$, therefore, the two processes have probabilities of occupying state $i$ at time $n$ which differ by at most $\varepsilon$, and this proves (2.9). It is also easy to see from (2.3) and Lemma 2.1 that $n$ can be chosen uniformly in $x^{\prime}$ and $x^{\prime \prime}$.

With this much preparation we shall now prove the first theorem:

\section{Theorem 2.1. The quantities}

$$
\lim _{n \rightarrow \infty} p_{i}^{(n)}(x)=\pi_{i}
$$

exist, are independent of $x$, and satisfy $\sum_{1} \pi_{i}=1$; the convergence is uniform in $x$.

Proof. Applying (2.2) repeatedly, we have

$$
\begin{aligned}
p_{i}^{(n+m)}(x) & =\sum_{x_{m}} p_{i_{m-1}}(x) p_{i_{m-2}}\left(i_{m-1}+x\right) \cdots p_{i_{0}}\left(i_{1}+\cdots+i_{m-1}+x\right) p_{i}^{(n)}\left(x_{m}+x\right)
\end{aligned}
$$

where $x_{m}=i_{0}, i_{1}, \cdots, i_{m-1}$. Therefore

$$
\begin{aligned}
& \left|p_{l}^{(n+m)}(x)-p_{i}^{(n)}(x)\right| \\
& \quad \leqq \sum_{x_{m}} p_{i_{m-1}}(x) \cdots p_{i_{0}}\left(i_{1}+\cdots+i_{m-1}+x\right)\left|p_{i}^{(n)}\left(x_{m}+x\right)-p_{i}^{(n)}(x)\right|
\end{aligned}
$$

and by Lemma 2.2, for any $\varepsilon$ there is an $n$ such that each term within absolute value signs on the right is less than $\varepsilon$. Since the weights $p_{i_{m-1}}(x) \cdots p_{i_{0}}\left(i_{1}+\cdots+i_{m-1}+x\right)$ sum to one, we have

$$
\left|p_{\imath}^{(n+m)}(x)-p_{i}^{(n)}(x)\right|<\varepsilon,
$$

and so $p_{i}^{(n)}(x)$ has a (uniform in $x$ ) limit $\pi_{i}$. Since there are a finite number of states,

$$
\sum_{i} \pi_{i}=\sum_{i} \lim _{n \rightarrow \infty} p_{i}^{(n)}(x)=\lim _{n \rightarrow \infty} \sum p_{i}^{(n)}(x)=1,
$$


and this completes the proof.

Next we shall define joint probabilities. If $x_{m}$ is $i_{0}, i_{1}, \cdots, i_{m-1}$, let

$$
\begin{aligned}
p_{x_{m}}\left(x^{\prime}\right) & =p_{x_{m}}^{(1)}\left(x^{\prime}\right) \\
& =p_{i_{m-1}}\left(x^{\prime}\right) p_{i_{m-2}}\left(i_{m-1}+x^{\prime}\right) \cdots p_{i_{0}}\left(i_{1}+\cdots+i_{m-1}+x\right) .
\end{aligned}
$$

This is, of course, the probabilitity of executing the sequence of states $x_{m}$ starting with past history $x^{\prime}$. We can define also the higher joint probabilities:

$$
p_{x_{m}}^{(n)}\left(x^{\prime}\right)=\sum_{j \in I} p_{j}\left(x^{\prime}\right) p_{x_{m}}^{(n-1)}\left(j+x^{\prime}\right) .
$$

Analogues of Lemmas 2.1 and 2.2 can be proved for these quantities by the same arguments used already; in this way it is not difficult to prove

\section{THEOREM 2.2. The quantities}

$$
\lim _{n \rightarrow \infty} p_{x_{m}}^{(n)}\left(x^{\prime}\right)=\pi_{x_{m}}
$$

exist, are independent of $x^{\prime}$, and satisfy $\sum_{i_{0}} \sum_{i_{m-1}} \pi_{x_{m}}=1$; the convergence is uniform in $x^{\prime}$.

REMARK. These two theorems imply the existence of a stationary stochastic process with the $p_{i}(x)$ for transition probabilities. The idea is that the quantities $\pi_{x_{m}}$ can be used to define a probability measure on the "cylinder sets" in the space of infinite sequences of members of $I$, and this measure can then be extended. This stationary process need not concern us further here.

Finally we will prove convergence theorems for certain "moments" which are useful in studying experimental data. The idea is that if we have a stochastic process with the functions $p_{i}(x)$ for transition probabilities, the probability $p_{i}\left(x_{m}\right)$ that the state at time $m$ is $i$ given the past history $x_{m}$ is itself a random variable, and so it makes sense to study $E\left(p_{i}^{\nu}\left(x_{m}\right)\right)$. More formally, define

$$
\alpha_{i}^{\nu}(m, x)=\sum_{i_{0}, \ldots, i_{m-1}} p_{i}^{\nu}\left(x_{m}+x\right) p_{x_{m}}(x)
$$

where $p_{x_{m}}(x)$ is defined by (2.11). Thus $\alpha_{i}^{1}(m, x)$ is the same as $p_{l}^{(m)}(x)$. Theorem 2.1 states that $\lim _{m \rightarrow \infty} \alpha_{i}^{1}(m, x)=\pi_{i}$ exists. We shall now prove

\section{THEOREM 2.3. The quantities}

$$
\lim _{m \rightarrow \infty} \alpha_{i}^{\nu}(m, x)=\alpha_{\imath}^{\nu}
$$

exist for every positive integer $\nu$; convergence is uniform in $x$ and the limit is independent of $x$. 
Proof. We use a simple estimate to show that $\alpha_{i}^{\nu}(m, x)$ is a Cauchy sequence:

$$
\begin{aligned}
& \left|\alpha_{i}^{\nu}(m+k+h, x)-\alpha_{i}^{\nu}(m+k, x)\right| \\
& \quad=\left|\sum_{x_{m+k+h}} p_{i}^{\nu}\left(x_{m+k+h}+x\right) p_{x_{m+k+h}}(x)-\sum_{x_{m+k}} p_{i}^{\nu}\left(x_{m+k}+x\right) p_{x_{m+k}}(x)\right| \\
& \quad \leqq \sum_{x_{m+k+h}}\left|p_{i}^{\nu}\left(x_{m+k+h}+x\right)-p_{l}^{\nu}\left(x_{m}+x\right)\right| p_{x_{m+k+h}}(x) \\
& \quad+\sum_{x_{m+k}}\left|p_{i}^{\nu}\left(x_{m+k}+x\right)-p_{i}^{\nu}\left(x_{m}+x\right)\right| p_{x_{m+k}}(x) \\
& \quad+\sum_{x_{m+k+h}} p_{i}^{\nu}\left(x_{m}+x\right) p_{x_{m+k+h}}(x)-\sum_{x_{m+k}} p_{i}^{\nu}\left(x_{m}+x\right) p_{x_{m+k}}(x) \mid .
\end{aligned}
$$

If $m$ is chosen large enough, the first two terms will be arbitrarily small; this involves nothing more than the conditions (resulting from (2.3) and (2.5)) that $\varepsilon_{m} \rightarrow 0$, and that a long sequence $x$ contains $j_{0}$ many times with high probability. The last term may be rewritten by carrying out the summation over all the indices except those in $x_{m}$; this yields

$$
\left|\sum_{x_{m}} p_{i}^{\curlyvee}\left(x_{m}+x\right)\left(p_{x_{m}}^{(k+h)}(x)-p_{x_{m}}^{(k)}(x)\right)\right| \leqq \sum_{x_{m}}\left|p_{x_{m}}^{(k+h)}(x)-p_{x_{m}}^{(k)}(x)\right|
$$

which is small for all $h$ (and for all $x$ ) if $k$ is large enough, by Theorem 2.2. Thus if $n=m+k,\left|\alpha_{i}^{\nu}(n+h, x)-\alpha_{i}^{\nu}(n, x)\right|$ is small for all $h$, and this proves that the limit (2.15) must exist; the limit is uniform in $x$ since $\alpha_{i}^{\nu}(m, x)$ is uniformly Cauchy. Another estimate along much the same line can be made to show that for any $\varepsilon>0$,

$$
\left|\alpha_{i}^{\nu}(m+k, x)-\alpha_{i}^{\nu}\left(m+k, x^{\prime}\right)\right| \leqq \varepsilon
$$

provided $m$ and $k$ are large. Since the limit of $\alpha_{i}^{\nu}(m+k, x)$ exists as $m+k \rightarrow \infty$, we can conclude that the limit is the same for all $x$.

It is also desirable to consider some additional "cross" moments involving $p_{i}\left(x_{m}\right)$ for several states at once; accordingly we define

$$
\alpha_{j_{1} j_{2} \cdots j_{k}}^{\nu_{1} \nu_{2} \cdots \nu_{k}}(m, x)=\sum_{x_{m}} p_{j_{1}}^{\nu_{1}}\left(x_{m}+x\right) p_{j_{2}}^{\nu_{2}}\left(x_{m}+x\right) \cdots p_{j_{k}}^{\nu_{k}}\left(x_{m}+x\right) p_{x_{m}}(x) \text {. }
$$

The following theorem is then a generalization of Theorem 2.3, which treats the case $k=1$ :

\section{THEOREM 2.4. The quantities}

$$
\lim _{m \rightarrow \infty} \alpha_{j_{1} \cdots j_{k}}^{\nu_{1} \cdots \nu_{k}}(m, x)=\alpha_{j_{1} \cdots \nu_{k}}^{\nu_{1} \cdots \nu_{k}}
$$

exist uniformly in $x$ for all non-negative integers $\nu_{1} \cdots \nu_{k}$ and all $j_{1} \cdots j_{k} \in I$, and the limits are independent of $x$.

The argument used in proving Theorem 2.3 works in this case also with only trivial changes, and need not be repeated. Finally we remark that moments involving several values of $n$ can be considered, and it 
can be shown that their limits exist also. This provides a generalization of Theorem 2.2.

3. Definition of linear learning models. The models we consider apply to an experimental situation which consists of a sequence of trials. On each trial the subject of the experiment makes a response, which is followed by a reinforcing event. Thus an experiment may be represented by a sequence $\left(A_{1}, E_{1}, A_{2}, E_{2}, \cdots A_{n}, E_{n}, \cdots\right)$ of random variables, where the choice of letters follows conventions established in the literature: the value of the random variable $A_{n}$ is a number $j$ representing the actual response on trial $n$, and the value of $E_{n}$ is a number $k$ representing the reinforcing event on trial $n$. The relevant data on each trial may then be represented by an ordered pair $(j, k)$ of integers with $1 \leqq j \leqq r$, and $0 \leqq k \leqq t$, that is, we envisage in general $r$ responses and $t+1$ reinforcing events. Any sequence of these pairs of integers is a sequence of values of the random variables and thus represents a possible experimental outcome. The general aim of the theory is to predict the probability distribution of the response random variable when a particular distribution, or class of distributions, is imposed on the reinforcement random variable.

In dealing with the general linear model with $r$ responses and $t+1$ reinforcing events we are following the formulation in Chapter 1 of Bush and Mosteller [2], although our notation is somewhat different, being closer to Estes [4] and Estes and Suppes [6].

The theory is formulated for the probability of a response on trial $n+1$ given the entire preceding sequence of responses and reinforcements. For this preceding sequence we use the notation $x_{n}$. Thus

$$
x_{n}=\left(k_{n}, j_{n}, k_{n-1}, j_{n-1}, \cdots, k_{1}, j_{1}\right) .
$$

(It is convenient to write these sequences in this order, but note that the numbering here is from past to present, not the reverse as in $\S 2$.) Our single axiom is the following linearity assumption:

Axiom $L$. If $E_{n}=k$ and $P\left(x_{n}\right)>0$ then

$$
P\left(A_{n+1}=j \mid x_{n}\right)=\left(1-\theta_{k}\right) P\left(A_{n}=j \mid x_{n-1}\right)+\theta_{k} \lambda_{j k},
$$

where $0 \leqq \theta_{k}, \lambda_{j k} \leqq 1$ and $\sum_{j} \lambda_{j k}=1$.

We obtain the linear model studied intensitively in [6] by setting:

$$
\left\{\begin{array}{rlrl}
\theta_{k} & =\theta & & \text { for } k \neq 0 \\
\theta_{k} & =0 & & \text { for } k=0 \\
\lambda_{j j} & =1 & & \\
\lambda_{j k} & =0 & & \text { for } j \neq k \\
t & =r . &
\end{array}\right.
$$


A linear model satisfying (3.2) we shall term an Estes Model, and for such models (3.1) may be replaced by the simpler condition:

(3.3) $P\left(A_{n+1}=j \mid x_{n}\right)= \begin{cases}(1-\theta) P\left(A_{n}=j \mid x_{n-1}\right)+\theta & \text { if } E_{n}=j \\ (1-\theta) P\left(A_{n}=j \mid x_{n-1}\right) & \text { if } E_{n}=k, k \neq 0, k \neq j \\ P\left(A_{n}=j \mid x_{n-1}\right) & \text { if } E_{n}=0 .\end{cases}$

Axiom $L$ satisfies the combining classes condition of Bush and Mosteller. Upon replacing $\theta$ by $1-\alpha$ in (3.1) essentially their general formulation of the linear model is obtained, although they do not explicitly indicate dependence on the sequence $x_{n}$.

We also define here certain moments which are of experimental interest and whose asymptotic properties we investigate subsequently. The moments $\alpha_{j, n}^{\prime}$ of the response probabilities at trial $n$ are:

$$
\alpha_{j, n}^{\nu}=\sum_{x_{n-1}} P^{\nu}\left(A_{n}=j \mid x_{n-1}\right) P\left(x_{n-1}\right) .
$$

And if the appropriate limits exist, we define

$$
\alpha_{j}^{\nu}=\lim _{n \rightarrow \infty} \alpha_{j, n}^{\nu} .
$$

The moments (3.4) are formed in an unsymmetrical way; however, they enter in a natural way in the expression of quantities which are easily observed experimentally-for instance, the joint probability $P\left(A_{n+1}=j, A_{n}=j\right)$. (For other examples, see [6].)

We are also interested in studying extensions of the linear model to multiperson situations. We may suppose that we have $s$ subjects in a situation such that the probability of a particular reinforcing event for any one subject will depend in general on preceding responses and reinforcements of the other $s-1$ subjects as well as on his own prior responses and reinforcements. The data on each trial may then be represented by an ordered $2 s$-tuple $\left(j_{1}, k_{1}, \cdots, j_{s}, k_{s}\right)$ of integers with $1 \leqq j_{i} \leqq r_{i}, 0 \leqq k_{i} \leqq t_{i}$, for $i=1, \cdots, s$, and any sequence of such tuples represents a possible experimental outcome. Let $A_{n}^{(i)}$ and $E_{n}^{(i)}$ be the response and reinforcement random variables for the $i$ th subject on trial $n$. We may then generalize Axiom $L$ to:

Axiom $M$. For $1 \leqq i \leqq s$, if $E_{n}^{(i)}=k$ and $P\left(x_{n}\right)>0$ then

$$
P\left(A_{n+1}^{(i)}=j \mid x_{n}\right)=\left(1-\theta_{\nu}^{(i)}\right) P\left(A_{n}^{(i)}=j \mid x_{n-1}\right)+\theta_{k}^{(i)} \lambda_{j k}^{(i)},
$$

where $0 \leqq \theta_{k}^{(i)}, \lambda_{j k}^{(i)} \leqq 1$ and $\sum_{j} \lambda_{j k}^{(i)}=1$.

Experimental tests of Axiom $M$ for two-person situations are reported in Estes [5] and in Atkinson and Suppes [1]. Let $x_{n-1}^{(i)}$ be just the 
sequence of first $n-1$ responses and reinforcements of subject $i$. It is a consequence ${ }^{1}$ of Axiom $M$ that

$$
P\left(A_{n}^{(i)}=j \mid x_{n-1}^{(i)}\right)=P\left(A_{n}^{(i)}=j \mid x_{n-1}\right),
$$

and it is in terms of $x_{n-1}^{(i)}$ that we define moments $\alpha_{\nu, j, n}^{(i)}$ exactly analogous to (3.4). We shall also be interested in the joint moments

$$
\gamma_{j_{1}, \cdots j_{s}, n}^{\nu}=\sum_{x_{n-1}} P^{\prime}\left(A_{n}^{(1)}=j_{1}, \cdots, A_{n}^{(s)}=j_{s} \mid x_{n-1}\right) P\left(x_{n-1}\right),
$$

and their asymptotes $\gamma_{j_{1}, \ldots, j_{s}}^{\nu}$ if they exist. To work with these latter moments in terms of Axiom $M$ we need the additional reasonable assumption that when all the $n-1$ preceding responses and reinforcements are given, the $s$ responses on trial $n$ are statistically independent:

Axiom I. If $P\left(x_{n-1}\right)>0$ then

$$
P\left(A_{n}^{(1)}=j_{1}, \cdots, A_{n}^{(s)}=j_{s} \mid x_{n-1}\right)=\prod_{i=1}^{s} P\left(A_{n}^{(i)}=j_{i} \mid x_{n-1}\right) .
$$

The experimental restriction implied by Axiom $I$ has been satisfied in the multiperson studies employing the linear model.

4. Asymptotic theorems for learning models. After dealing with some matters of notation, we state general theorems on the existence of asymptotic moments. The hypotheses of the theorems give some broad conditions which guarantee ergodic behavior. We begin with the one-person models satisfying Axiom $L$.

In this section it will be convenient to use some of the notation of §2. Thus we may write $P\left(A_{n}=j \mid x_{m}+x^{\prime}\right)$ in place of $P\left(A_{n}=j \mid x_{n-1}\right)$ to indicate we are interested in the last $m$ terms of $x_{n-1}$. The "sum" $x_{m}+x^{\prime}$ is just the combined sequence $x_{n-1}$. We reserve the subscript $m$ for counting back $m$ trials from a given trial $n$.

To clarify the general theorem it is desirable to define in an exact way the notion of the conditional probability of a reinforcing event depending on only a finite number $m$ of past trial outcomes and independent of the trial number.

DEFINITION. A linear model has a reinforcement schedule with past dependence of length $m$ if, and only if, for all $k, n$ and $n^{\prime}$ with $n, n^{\prime}>m$ and all $x_{m}, x^{\prime}$ and $x^{\prime \prime}$

$$
P\left(E_{n}=k \mid x_{m}+x^{\prime}\right)=P\left(E_{n}=k \mid x_{m}+x^{\prime \prime}\right) .
$$

(It is understood that $x_{m}$ includes the response $A_{j, n}$ which precedes $E_{k, n}$ on trial $n$.) It is to be noticed that the use of $n$ on one side and $n^{\prime}$ on the other side of (4.1) yields independence of trial number. The term

\footnotetext{
1 Proof of this fact is analogous to that of Theorem 4.8 of [6].
} 
reinforcement schedule has been used because of its frequent occurrence with approximately this meaning in the experimental literature. For the conditional probabilities of (4.1) we shall use the notation

$$
\pi_{k, x_{m}}=P\left(E_{n}=k \mid x_{m}+x\right) .
$$

We may now state the first general theorem.

THEOREM 4.1. Let $\mathscr{P}$ be a linear model such that

(i) $\mathscr{L}$ has a reinforcement schedule with past dependence of length $m^{*}$,

(ii) there is an integer $k^{*}$ such that

(a) $\theta_{k^{*}} \neq 0$

(b) there is a $\delta^{*}$ and an $m_{0}$ such that for all sequences $x$ and all integers $n$

$$
P\left(E_{n+m_{\mathrm{J}}}=k^{*} \mid x_{n}\right) \geqq \delta^{*}>0 .
$$

Then the asymptotic moments $\alpha_{j}^{\nu}$ of $\mathscr{L}$ all exist and are independent of the initial distribution of responses.

Proof. The central task is to characterize $\mathscr{L}$ as a chain of infinite order and show that satisfaction of the hypotheses of the theorem implies satisfaction of conditions (2.3) and (2.5). With this accomplished the asymptotic theorems of $\$ 2$ may be applied to $\mathscr{C}$. It is most convenient to take as states of the chain the ordered pairs $(j, k)$, where $j$ is the response on trial $n$, say, and $k$ is the reinforcement on the preceding trial. Consider now the reinforcement $k^{*}$ of the hypothesis of the theorem. Let $j^{*}$ be a response such that $\lambda_{s^{*} k^{*}} \neq 0$. (There is at least one such $j^{*}$ since $\sum_{j} \lambda_{j k}=1$; in the Estes model $j^{*}=k^{*}$.) With the pair $\left(j^{*}, k^{*}\right)$ as the state $j_{0}$ of the infinite order chain, we shall establish (2.3) and (2.5).

To verify (2.3), we use (ii)b of the hypothesis and the following equalities and inequalities, which hold for all $x$ and $n$ :

$$
\begin{aligned}
& P\left(A_{n+m_{0}+1}=j^{*}, E_{n+m_{0}}=k^{*} \mid x_{n}\right) \\
& =\sum_{x_{m_{0}-1}} P\left(A_{n+m_{0}+1}=j^{*} \mid E_{n+m_{0}}=k^{*}, x_{m_{0}-1}+x_{n}\right) \\
& \cdot P\left(E_{n+m_{0}} \mid x_{m_{0}-1}+x_{n}\right) P\left(x_{m_{0}-1} \mid x_{n}\right) .
\end{aligned}
$$

Applying Axiom, $L$, the right-hand side becomes:

$$
\begin{aligned}
& =\sum_{x_{m_{0}-1}}\left[\left(1-\theta_{k^{*}}\right) P\left(A_{n+m_{0}}=j^{*} \mid x_{m_{0}-1}+x_{n}\right)+\theta_{k^{*}} \lambda_{j^{*} k^{*}}\right] \\
& \text { - } P\left(E_{n+m_{0}}=k^{*} \mid x_{m_{0}-1}+x_{n}\right) \cdot P\left(x_{m_{0}-1} \mid x_{n}\right) \\
& \geqq \theta_{k^{*}} \lambda_{j^{*} k^{*}} \sum_{x_{m_{0}-1}} P\left(E_{n+m_{0}}=k^{*} \mid x_{m_{0}-1}+x_{n}\right) P\left(x_{m_{0}-1} \mid x_{n}\right) \\
& \geqq \theta_{k^{*}} \lambda_{j^{*} k^{*}} P\left(E_{n+m_{0}}=k^{*} \mid x_{n}\right) \\
& \geqq \theta_{k^{*}} \lambda_{j^{*} k_{*}} \delta^{*}
\end{aligned}
$$


To establish (2.5), consider the following equalities and inequalities:

$$
\begin{gathered}
\left|P\left(A_{n \prime+1}=j, E_{n \prime}=k \mid x+x^{\prime}\right)-P\left(A_{n \prime \prime+1}=j, E_{n \prime \prime}=k \mid x+x^{\prime \prime}\right)\right| \\
=\pi_{k, x^{*}}\left|P\left(A_{n \prime+1}=j \mid E_{n \prime}=k, x+x^{\prime}\right)-P\left(A_{n \prime \prime+1}=j \mid E_{n \prime \prime}=k, x+x^{\prime \prime}\right)\right|,
\end{gathered}
$$

where $x_{m^{*}}$ means the last $m^{*}$ terms of $x$, and where the sequence $x$ contains at least $m$ occurrences of $k^{*}$, with $m>m^{*}$. The equality follows from (i) of the hypothesis, for by virtue of (i)

$$
\pi_{k, m^{*}}=P\left(E_{n \prime}=k \mid x+x^{\prime}\right)=P\left(E_{n \prime \prime}=k \mid x+x^{\prime \prime}\right) .
$$

Applying Axiom $L$ once to the right-hand side of (4.3) we get, ignoring $\pi_{k, x_{m}}$ :

$$
\begin{aligned}
& \left|P\left(A_{n \prime+1}=j \mid E_{n \prime}=k, x+x^{\prime}\right)-P\left(A_{n \prime \prime+1}=j \mid E_{n \prime \prime}=k, x+x^{\prime \prime}\right)\right| \\
& \quad=\left(1-\theta_{k}\right)\left|P\left(A_{n \prime}=j \mid x+x^{\prime}\right)-P\left(A_{n \prime \prime}=j \mid x+x^{\prime \prime}\right)\right| .
\end{aligned}
$$

We do not know that $\theta_{k} \neq 0$, but as we apply Axiom $L$ repeatedly, we obtain the factor $\left(1-\theta_{k^{*}}\right)$ at least $m$ times, so that

$$
\begin{aligned}
& \left|P\left(A_{n^{\prime}+1}=j, E_{n \prime}=k \mid x+x^{\prime}\right)-P\left(A_{n \prime \prime+1}=j, E_{n \prime \prime}=k \mid x+x^{\prime \prime}\right)\right| \\
& \quad \leqq\left(1-\theta_{k^{*}}\right)^{m}\left|P\left(A_{n \prime-h}=j \mid x^{\prime}\right)-P\left(A_{n \prime \prime-h} \mid x^{\prime \prime}\right)\right|,
\end{aligned}
$$

where $h$ is the length of $x^{2}$. The difference term on the right of this inequality is not more than 1 , so that from (4.4) we obtain the estimate for $m>m^{*}$

$$
\varepsilon_{m} \leqq\left(1-\theta_{k^{*}}\right)^{m}
$$

whence

$$
\sum_{m=0}^{\infty} \hat{\varepsilon}_{m}<\infty,
$$

which is (2.5).

On the basis of (2.3) and (2.5) we know from Theorem 2.4 that the asymptotic cross-moments of $\mathscr{L}$ exist and are independent of the initial distribution of responses. But

$$
P\left(A_{n}=j \mid x_{n-1}\right)=\sum_{k} P\left(A_{n}=j, E_{n-1}=k \mid x_{n-1}\right),
$$

and so the moments $\alpha_{j, n}^{\nu}$ can be expressed as sums of the cross-moments for the infinite order chain $\mathscr{L}$, which insures the existence of the limitting moments (3.5) and that they do not depend upon initial conditions.

There are several remarks to be made about the theorem just

2 If all $\theta_{k} \neq 0$, the original condition given in [3] would be satisfied; our weaker condition (2.5) allows inclusion of cases where some of the $\theta_{k}$ are 0 (i.e. where there can be trials without a reinforcement). 
proved. First, we observe that a simple sufficient (but not necessary) condition for (ii)b is

$$
\min _{x_{m^{*}}} \pi_{k^{*}, x_{m^{*}}} \neq 0
$$

The interpretation of (4.5) is that the reinforcing event $k^{*}$ has positive probability on every trial no matter what sequence $x_{m^{*}}$ of responses and reinforcements preceded. A number of interesting experimental cases of the linear model can be described in terms of (4.5), (i) and (ii)a of Theorem 4.1.

I. Contingent case with lag $v$. In the Estes model let $P\left(E_{n}=\right.$ $\left.k \mid A_{n-v}=j, x\right)=\pi_{k j}(v)$, for all $x$ such that $P\left(A_{n-v}=j, x\right)>0$. To satisfy (4.5), we need only that for some $k, \pi_{j k}(v) \neq 0$ for all $j$. Experimental data for $v=0,1,2$ are given in Estes [5].

II. Double contingent case. Let

$$
P\left(E_{n}=k \mid A_{n}=j, A_{n-1}=j^{\prime}, x\right)=\pi_{k, j \jmath \prime},
$$

for all $x$ such that $P\left(A_{n}=j, A_{n-1}=j^{\prime}, x\right)>0$.

Then (i) of Theorem (4.1) is immediately satisfied, and for (ii)a and (4.5) we need a $k$ such that $\theta_{k} \neq 0$ and for all $j$ and $j^{\prime}, \pi_{k, j j^{\prime}} \neq 0$.

An interesting fact about (I) and (II) is that although they are simple to test experimentally and their asymptotic response moments exist on the basis of Theorem 4.1, there is no known constructive method for computing the actual asymptotes. (The Estes [5] test of (I) excludes non-reinforced trials which cause the computational difficulties.) It may also be noted that the convergence theorems in Karlin [8] do not in general apply to (II), and apply to (I) only if $v=0$.

On the basis of the proof of Theorem 4.1 we may, by virtue of Theorem 2.2, conclude that the asymptotic joint probabilities of successive responses also exist:

COROLlary 1. If the hypothesis of Theorem 4.1 is satisfied, then for every $m$ the limit as $n \rightarrow \infty$ of

$$
P\left(A_{n+m}=j_{m}, A_{n+m-1}=j_{m-1}, \cdots, A_{n}=j_{0}\right)
$$

exists.

We may regard the quantities $P\left(A_{n}=j \mid x_{n-1}\right)$, for $1 \leqq j \leqq r$ as a random probability vector with an arbitrary joint distribution $F_{1}$ on trial 1 , and distribution $F_{n}$ on trial $n$. The following corollary is a consequence of the existence of the moments $\alpha_{j}^{v}$ independent of the initial response probabilities. 
COROLlary 2. If the hypothesis of Theorem 4.1 is satisfied, then there is a unique asymptotic distribution $F_{\infty}$, independent of $F_{1}$ to which the distributions $F_{n}$ converge.

For the multiperson situation characterized by Axioms $I$ and $M$, we have a theorem analogous to Theorem 4.1. For use in the hypothesis of this theorem we define the notion of reinforcement schedule with past dependence of length $m$, exactly as we did in (4.1), namely, we have such a schedule if for all $k, 1 \leqq i \leqq s$, all $n$ and $n^{\prime}$ with $n, n^{\prime}>m$ and all $x_{m}, x^{\prime}$ and $x^{\prime \prime}$

$$
\begin{aligned}
\pi_{k(1), \cdots, k_{k}^{(s),} x_{m}} & =P\left(\underline{E}_{n}^{(1)}=k^{(1)}, \cdots, \underline{E}_{n}^{(s)}=k^{(s)} \mid x_{m}+x^{\prime}\right) \\
& =P\left(E_{n}^{(1)}=k^{(1)}, \cdots, E_{n}^{(s)}=k^{(s)} \mid x_{m}+x^{\prime \prime}\right) .
\end{aligned}
$$

THEOREM 4.2. Let Ll be an s-person linear model such that

(i) Il has a reinforcement schedule with past dependence of length $m^{*}$,

(ii) there are integers $k^{(i)^{*}}$, for $1 \leqq i \leqq s$, such that

(a) $\theta_{k^{(i) *}}^{(i)} \neq 0$

(b) there is $a \delta^{*}$ and an $m_{0}$ such that for all sequences $x$ and all integers $n$

$$
P\left(E_{n+m_{0}}^{(1)}=k^{(1)^{*}}, \cdots, E_{n+m_{0}}^{(s)}=k^{(s)^{*}} \mid x_{n}\right) \geqq \delta^{*}>0 .
$$

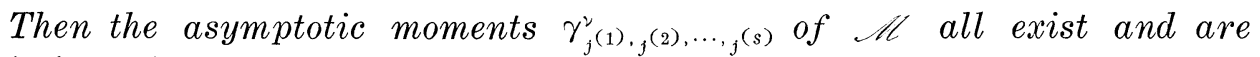
independent of the initial distribution of responses.

Proof. The states of the chain are now defined as $2 s$-tuples $\left(i^{(1)}, \cdots, j^{(s)}, k^{(1)}, \cdots, k^{(s)}\right)$, where $j^{(i)}$ is the response made by the $i$ th subject and $k^{(i)}$ is the reinforcement for that subject on the preceding trial. Using the reinforcements $k^{(i)^{*}}$ of the hypothesis, let $j^{(i)^{*}}$ be such that $\lambda_{j^{(i) *} k_{k}^{(i)^{*}}}^{(i)} \neq 0$. We take $\left(j^{(1)^{*}}, \cdots, j^{(s)^{*}}, k^{(1)^{*}}, \cdots, k^{(s)^{*}}\right)$ as the state $j_{0}$ for which we establish (2.3) and (2.5). To simplify notation, it is convenient to define:

$$
\begin{aligned}
& P_{n+1}(j, k \mid x)=P\left(A_{n+1}^{(1)}=j^{(1)}, \cdots, A_{n+1}^{(s)}=j^{(s)}, E_{n}^{(1)}=k^{(1)}, \cdots, E_{n}^{(s)}=k^{(s)} \mid x\right), \\
& p_{n+1}\left(j^{(i)} \mid k, x\right)=P\left(A_{n+1}^{(i)}=j^{(i)} \mid E_{n}^{(1)}=k^{(1)}, \cdots, E_{n}^{(s)}=k^{(s)}, x\right), \\
& \pi_{k, m^{*}}=\pi_{k}^{(1), \cdots,{ }_{k}(s) *},{ }_{x^{*}} .
\end{aligned}
$$

Moreover, we omit the superscript notation from $\theta$ and $\lambda$.

To verify (2.3) we proceed exactly as in the proof of Theorem 4.1, applying now Axioms $I$ and $M$ instead of $L$, and we obtain that

$$
p_{n+m_{0}+1}\left(j, k \mid x_{n}\right) \geqq \prod_{i=1}^{s} \theta_{k}^{(i) * \lambda_{j}(i) *{ }_{k}(i) *} \delta^{*} .
$$


For (2.5), we first observe that by virtue of (i) of the hypothesis and Axiom $I$

$$
\begin{aligned}
& \left|p_{n^{\prime}+1}\left(j, k \mid x+x^{\prime}\right)-p_{n^{\prime \prime+1}}\left(j, k \mid x+x^{\prime \prime}\right)\right| \\
& \quad=\pi_{k, m *}\left|\prod_{i=1}^{s} p_{n^{\prime}+1}\left(j^{(i)} \mid k, x+x^{\prime}\right)-\prod_{i=1}^{s} p_{n^{\prime \prime+1}}\left(j^{(i)} \mid k, x+x^{\prime \prime}\right)\right| .
\end{aligned}
$$

We notice next that the right-hand side is

$$
\begin{aligned}
\leqq & \pi_{k, m^{*}}\left\{p_{n^{\prime}+1}\left(j^{(1)} \mid k, x+x^{\prime}\right) \mid \prod_{i=2}^{s} p_{n^{\prime}+1}\left(j^{(i)} \mid k, x+x^{\prime}\right)\right. \\
& -\prod_{i=2}^{s} p_{n^{\prime \prime}+1}\left(j^{(i)} \mid k, x+x^{\prime \prime}\right) \mid \\
& +\prod_{i=2}^{s} p_{n^{\prime \prime}+1}\left(j^{(i)} \mid k, x+x^{\prime \prime}\right)\left|p_{n^{\prime}+1}\left(j^{(1)} \mid k, x+x^{\prime}\right)-p_{n^{\prime \prime+1}}\left(j^{(1)} \mid k, x+x^{\prime \prime}\right)\right| .
\end{aligned}
$$

Continuing this same development, we obtain:

$$
\leqq \sum_{i=1}^{s}\left|p_{n^{\prime}+1}\left(j^{(i)} \mid k, x+x^{\prime}\right)-p_{n^{\prime \prime}+1}\left(j^{(i)} \mid k, x+x^{\prime \prime}\right)\right| \text {. }
$$

And by the line of reasoning used in the proof of Theorem 4.1, if the sequence $x$ contains state $\left(j^{(1)^{*}}, \cdots, k^{(s)^{*}}\right)$ at least $m$ times the last quantity is

$$
\leqq \sum_{i=1}^{s}\left(1-\theta_{k}(i) *\right)^{m}
$$

Provided $m>m^{*}$ this inequality yields an estimate of $\varepsilon_{m}$ from which we conclude that (2.5) holds. The existence of the asymptotic moments then follows from the theory of $\S 2$ as in the case of Theorem 4.1. Q.E.D.

A pair of corollaries follow from the theorem just proved which are exactly like the two given after Theorem 4.1.

Finally, we want to remark that Axiom $L$ involves linear functions which are distance diminishing, i.e., have slope less than one. The asymptotic results of this section apply to many learning models in which these linear functions are replaced by non-linear functions having this property.

\section{REFERENCES}

1. Richard C. Atkinson, and Patrick Suppes, An analysis of iwo-person game situations in terms of statistical learning theory, J. of Experimental Psychology, 55 (1958), 369-378. 2. Robert R. Bush, and Frederick Mosteller, Stochastic Models for Learning, New York, 1955.

3. W. Doeblin, and R. Fortet, Sur des chaînes à liaisons complètes, Bull. Soc. Math. France, 65 (1937), 132-148.

4. W. K. Estes, Theory of learning with constant, variable, or contingent probabilities of reinforcement, Psychometrika, 22 (1957), 113-132.

5. —_, Of models and men, Amer. Psychologist, 12 (1957), 609-617. 
6. W. K. Estes, and Patrick Suppes, Foundarions of Statistical Learning Theory, I. The Linear Model for Simple Learning, Technical Report No. 16, Contract Nonr 225(17), Applied Mathematics and Statistics Laboratory, Stanford University, 1957. An abridged version appears as Chapter 8 of Studies in Mathematical Learning Theory, edited by R. R. Bush and W. K. Estes, Stanford University Press, 1959.

7. T. E., Harris, On chains of of infinite order, Pacific J. Math., 5 (1955), 707-724.

8. Samuel Karlin, Some random walks arising in learning models I, Pacific J. Math., 3 (1953), 725-756.

9. Maurice Kennedy, A convergence theorem for a certain class of Markoff processes, Pacific J. Math., 7 (1957), 1107-1124.

STANFORD UNIVERSITY 


\section{PACIFIC JOURNAL OF MATHEMATICS}

\section{EDITORS}

\section{David Gilbarg}

Stanford University Stanford, California

\section{R. A. Beaumont}

University of Washington

Seattle 5, Washington

\section{A. L. Whiteman}

University of Southern California Los Angeles 7, California

L. J. Paige

University of California

Los Angeles 24, California

\section{ASSOCIATE EDITORS}

E. F. BECKENBACH
C. E. BURGESS
E. HEWITT
A. HORN

A. HORN

\author{
V. GANAPATHY IYER \\ R. D. JAMES \\ M. S. KNEBELMAN \\ L. NACHBIN
}

I. NIVEN

T. G. OSTROM

H. L. ROYDEN

M. M. SCHIFFER
E. G. STRAUS

G. SZEKERES

F. WOLF

K. YOSIDA

\section{SUPPORTING INSTITUTIONS}

\author{
UNIVERSITY OF BRITISH COLUMBIA \\ CALIFORNIA INSTITUTE OF TECHNOLOGY \\ UNIVERSITY OF CALIFORNIA \\ MONTANA STATE UNIVERSITY \\ UNIVERSITY OF NEVADA \\ OREGON STATE COLLEGE \\ UNIVERSITY OF OREGON \\ OSAKA UNIVERSITY \\ UNIVERSITY OF SOUTHERN CALIFORNIA
}

\author{
STANFORD UNIVERSITY \\ UNIVERSITY OF TOKYO \\ UNIVERSITY OF UTAH \\ WASHINGTON STATE COLLEGE \\ UNIVERSITY OF WASHINGTON \\ * * * * \\ AMERICAN MATHEMATICAL SOCIETY \\ CALIFORNIA RESEARCH CORPORATION \\ HUGHES AIRCRAFT COMPANY \\ SPACE TECHNOLOGY LABORATORIES
}

Mathematical papers intended for publication in the Pacific Journal of Mathematics should be typewritten (double spaced), and the author should keep a complete copy. Manuscripts may be sent to any one of the four editors. All other communications to the editors should be addressed to the managing editor, L. J. Paige at the University of California, Los Angeles 24, California.

50 reprints per author of each article are furnished free of charge; additional copies may be obtained at cost in multiples of 50 .

The Pacific Journal of Mathematics is published quarterly, in March, June, September, and December. The price per volume (4 numbers) is $\$ 12.00$; single issues, $\$ 3.50$. Back numbers are available. Special price to individual faculty members of supporting institutions and to individual members of the American Mathematical Society: $\$ 4.00$ per volume; single issues, $\$ 1.25$.

Subscriptions, orders for back numbers, and changes of address should be sent to Pacific Journal of Mathematics, 2120 Oxford Street, Berkeley 4, California.

Printed at Kokusai Bunken Insatsusha (International Academic Printing Co., Ltd.), No. 6, 2-chome, Fujimi-cho, Chiyoda-ku, Tokyo, Japan.

PUBLISHED BY PACIFIC IOURNAL OF MATHEMATICS, A NON-PROFIT CORPORATION

The Supporting Institutions listed above contribute to the cost of publication of this Journal, but they are not owners or publishers and have no responsibility for its content or policies. 


\section{Pacific Journal of Mathematics}

\section{Vol. 9, No. $3 \quad$ July, 1959}

Errett Albert Bishop, A minimal boundary for function algebras . . . . . . . . . . . . 629

John W. Brace, The topology of almost uniform convergence . . . . . . . . . . . . 643

Cecil Edmund Burgess, Chainable continua and indecomposability .......... 653

L. Carlitz, Multiplication formulas for products of Bernoulli and Euler

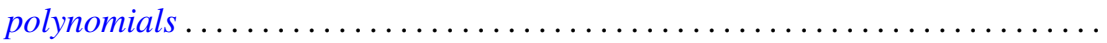

Eckford Cohen, A class of residue systems (mod $r$ ) and related arithmetical

functions. II. Higher dimensional analogues ....................

Shaul Foguel, Boolean algebras of projections of finite multiplicity . . . . . . . . . .

Richard Robinson Goldberg, Averages of Fourier coefficients .................

Seymour Goldberg, Ranges and inverses of perturbed linear operators .

Philip Hartman, On functions representable as a difference of convex functions ....

Milton Vernon Johns, Jr. and Ronald Pyke, On conditional expectation and

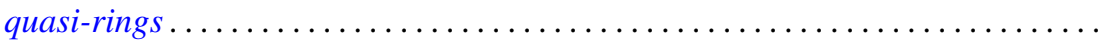

Robert Jacob Koch, Arcs in partially ordered spaces ....................

Gregers Louis Krabbe, A space of multipliers of type $L^{p}(-\infty, \infty) \ldots \ldots \ldots \ldots$

John W. Lamperti and Patrick Colonel Suppes, Chains of infinite order and their

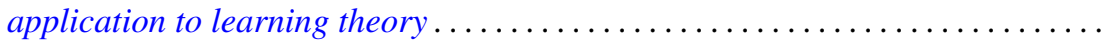

Edith Hirsch Luchins, On radicals and continuity of homomorphisms into Banach

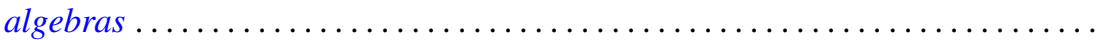

T. M. MacRobert, Multiplication formulae for the E-functions functions of their parameters.

Michael Bahir Maschler, Classes of minimal and representative domains and their kernel functions.

William Schumacher Massey, On the imbeddability of the real projective spaces in Euclidean space.

Thomas Wilson Mullikin, Semi-groups of class $\left(C_{0}\right)$ in $L_{p}$ determined by parabolic differential equations

Steven Orey, Recurrent Markov chains

Ernest Tilden Parker, On quadruply transitive groups ........ . .

Calvin R. Putnam, On Toeplitz matrices, absolute continuity, and unitary

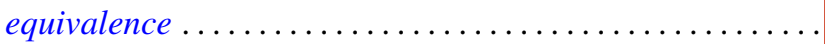

Helmut Heinrich Schaefer, On nonlinear positive operators.

Robert Seall and Marion Wetzel, Some connections between continued fractions and convex sets

Robert Steinberg, Variations on a theme of Chevalley

Olga Taussky and Hans Zassenhaus, On the similarity transformation between a

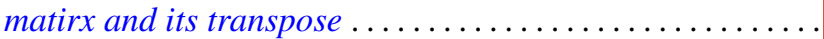

Emery Thomas, The suspension of the generalized Pontrjagin cohomology

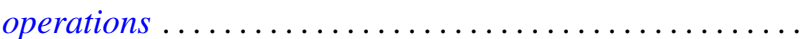

Joseph L. Ullman, On Tchebycheff polynomials ..................... 913

Richard Steven Varga, Orderings of the successive overrelaxation scheme ........ 925

Orlando Eugenio Villamayor, Sr., On weak dimension of algebras . 